\title{
Morphological Dynamics of Mitochondria in Bovine Aortic Endothelial Cell under Cyclic Stretch
}

\author{
Aya Shinmura, ${ }^{*}$ Akira Tsukamoto, ${ }^{*}, \#$ Tsuyoshi Hamada, ${ }^{*}$ Kouki Takemura, ${ }^{*}$ Takashi Ushida, ${ }^{* *}$ Shigeru Tada ${ }^{*}$
}

\begin{abstract}
Mitochondria are subcellular organelles that synthesize ATP, generate reactive oxygen species (ROS), and control cellular fates such as apoptosis and aging. Mitochondria generate different amounts of ROS in association with their morphologies. Cyclic stretch is a mechanical stimulation exerted on cells due to arterial pulsation, and induces cells to generate mitochondrial ROS. Therefore, one can speculate that morphological changes of mitochondria may play a role in mitochondrial ROS generation in cells under cyclic stretch. However, whether the morphologies of mitochondria are actually altered under cyclic stretch remains unclear. This study attempted to answer this question by time-lapse imaging the morphological dynamics of mitochondria in bovine aortic endothelial cells (BAECs) subjected to two levels of uniaxial cyclic stretch: (1) a physiologic level (5\% at $1 \mathrm{~Hz}$ ) for 1 hour, and (2) a supra-physiologic level (20\% at $1 \mathrm{~Hz})$ for 1 hour. Mitochondria were stained with Mito-tracker Orange, and MicroP software and FibrilTool were used for mitochondrial alignment and length analyses. No clear changes in the average length of mitochondria were observed at the physiological level of stretch (5\%) compared to no stretch $(0 \%)$, while the average length was decreased by the supra-physiological level of stretch (20\%). In addition, cellular alignment was not different between $0 \%$ and $5 \%$ stretches, but the cells became perpendicularly aligned in the direction of stretch when $20 \%$ stretch was applied. Cellular circularity was not significantly different among the three levels of cyclic stretch. Thus, BAECs exhibited changes in both mitochondrial dynamics and cellular remodeling dynamics under $20 \%$ stretch, but showed no changes in both under $5 \%$ stretch. The results indicate that changes in morphological dynamics of mitochondria correlate with changes in cellular dynamics, particularly change in cellular alignment.
\end{abstract}

Keywords: mitochondria, morphology, time-lapse imaging, bovine aortic endothelial cells, cyclic stretch.

Adv Biomed Eng. 4: pp. 60-66, 2015.

\section{Introduction}

Cyclic stretch is a mechanical stimulation that is exerted on endothelial cells due to arterial pulsation [1]. Under physiological elongation of $6-10 \%$, cyclic stretch induces anti-apoptotic effects $[2,3]$. On the other hand, under supra-physiological elongation of $20 \%$, cyclic stretch causes apoptosis $[2,4]$. The difference in response depending on the degree of elongation is thought to involve differences in the amounts of intracellular reactive oxygen species (ROS), superoxide $\left(\mathrm{O}_{2}{ }^{\bullet-}\right)$, and $\mathrm{H}_{2} \mathrm{O}_{2}[5,6]$. Because mitochondria produce ROS in cells under cyclic stretch [7, 8], mitochondria may be involved in the different cellular effects under physiological and supra-physiological cyclic stretches.

Mitochondria are subcellular organelles that synthesize ATP and play important roles in cellular signaling [9]. Mitochondria are dynamic organelles that undergo repeated fusion and fission $[10,11]$. When the balance of fusion and fission is disrupted, mitochondrial morphology is altered. In conjunction with these morphological alterations-particularly mitochondrial shorten-

This study was presented at the Symposium on Biomedical Engineering 2014, Tokyo, September, 2014.

Received on August 1, 2014; revised on October 1, 2014 and October 27, 2014; accepted on November 7, 2014.

* Department of Applied Physics, National Defense Academy of Japan, Yokosuka, Japan.

** Center for Disease Biology and Integrative Medicine, The University of Tokyo, Tokyo, Japan.

\# 1-10-20 Hashirimizu, Yokosuka, Kanagawa 239-8686, Japan.

E-mail: tsuka@nda.ac.jp ing - ROS production is increased [12]. Thus, morphological alterations of the mitochondria may be involved in mitochondrial ROS production under cyclic stretch.

The morphology of mitochondria depends on the cytoskeleton. Mitochondria are physically anchored to microtubules via adaptor molecules [13]. In that regard, the microtubules function as the backbone of mitochondria [14]. When the microtubules are disrupted, the morphological structures of the mitochondria are also disrupted [15]. In cells subjected to cyclic stretch, the alignment of cytoskeletons, especially actin, is altered so that the cell axis becomes perpendicular to the stretch axis [16]. Recently, alteration of their microtubule alignment has also been demonstrated [17]. Thus, it can be hypothesized that cyclic stretch alters the cytoskeletal alignment, thereby altering mitochondrial morphology and induces ROS production. Because alteration in cytoskeletal alignments has been shown to correlate with the elongation value of cyclic stretch [18], the hypothesis agrees with previous experimental finding that ROS production is enhanced under cyclic stretch with larger elongation values. These findings lead us to ask whether the morphologic alteration of mitochondria under cyclic stretch correlates with the elongation value of cyclic stretch.

In this study, in order to understand whether mitochondrial morphology is altered in cells under cyclic stretch, we time-lapse imaged mitochondria in bovine aortic endothelial cells (BAECs) under cyclic stretch. In addition, in order to understand whether the change in the mitochondrial morphology correlates with the elongation value of cyclic stretch, BAECs were subjected to two types of cyclic stretch, physiologic stretch causing 5\% elongation, and supra-physiologic stretch causing $20 \%$ elongation. 


\section{Methods}

\subsection{Cell culture}

BAECs (LONZA Japan Ltd.) were cultured in DMEM (Dulbecco's Modified Eagle's Medium; Sigma) supplemented with $10 \%$ fetal bovine serum, penicillin/streptomycin and L-glutamine. BAECs were cultured in a $60-\mathrm{mm}$ cell culture dish in a $5 \% \mathrm{CO}_{2}$ incubator at $37^{\circ} \mathrm{C}$ until they became confluent. For subculture, BAECs were detached with $0.025 \%$ trypsin solution. BAECs in the 3 rd to 7 th passage were used in experiments.

\subsection{Stretch chamber}

Base and curing agents of polydimethylsiloxane (PDMS) (Sylgard 184; Dow Corning Toray) were mixed in a ratio of 25:2 and poured into a handmade mold. For molding, PDMS was baked at $80^{\circ} \mathrm{C}$ for $1 \mathrm{~h}$ (Fig. 1A). The molded stretch chamber was connected to stretching motors by grippers (Fig. 1B). Before inoculating the cells in a stretch chamber, PDMS was treated with oxygen plasma (PDC-32G; Harrick Plasma) and coated with fibronectin by incubating with fibronectin solution $(20 \mu \mathrm{g} / \mathrm{ml}$ in PBS) at room temperature for 1 hour. The BAECs were then inoculated into the stretch chamber to $80 \%$ confluence, and further cultured overnight before the experiments.

\subsection{Cyclic stretch and fluorescence imaging}

To visualize the mitochondria, BAECs were stained with MitoTracker Orange ( $1 \mu \mathrm{M}$ in serum-free DMEM; Molecular Probes) in a $5 \% \mathrm{CO}_{2}$ incubator for $20 \mathrm{~min}$. After washing with fresh DMEM, the stretch chamber was connected to a stepper motor (SGSP-20-35; Sigma Koki). The BAECs were subjected to cyclic stretch to effect $5 \%$ or $20 \%$ elongation at $1 \mathrm{~Hz}$ frequency. Cyclic stretch was paused for 2 min every $10 \mathrm{~min}$ for fluorescence imaging of mitochondria (Fig. 2). During the pauses, fluorescence images were obtained from 6 imaging fields. The positions of the imaging fields were selected prior to cyclic stretch and preset in a PC so that the motorized XY stage on the microscope replicated all 6 positions. Fluorescence images were obtained using a confocal microscope (FV-1000; Olympus). For fluorescence imaging, exposures were made using excitation wavelength of $543 \mathrm{~nm}$ and emission wavelength of $576 \mathrm{~nm}$ through an objective lens $(60 \times$, N.A 1.1; Olympus). During cyclic stretch and fluorescence imaging, BAECs were incubated in a microscope incubator (Tokai Hit) with $5 \% \mathrm{CO}_{2}$ at $37^{\circ} \mathrm{C}$. BAECs were incubated in the microscope incubator for $30 \mathrm{~min}$ prior to cyclic stretch.

\subsection{Image analysis}

The fluorescence images obtained were trimmed with ImageJ (freehand selection and clear outside, NIH) to extract the images of a single cell. Brightness was further adjusted with ImageJ (brightness/contrast), and then the image size was standardized to $400 \times 400$ pixels using Irfan View (freeware) without changing the magnification.

For the evaluation and identification of mitochondrial morphology, the lengths and alignment angles of mitochondria were quantified from fluorescence images. For quantification of the length of mitochondria using MicroP software, a Matlab plugin developed by Peng et al. [19] specifically for the analysis of mitochondrial morphology was used. Although this program is a powerful tool for mitochondrial morphology analysis, it was not suit-
A

B
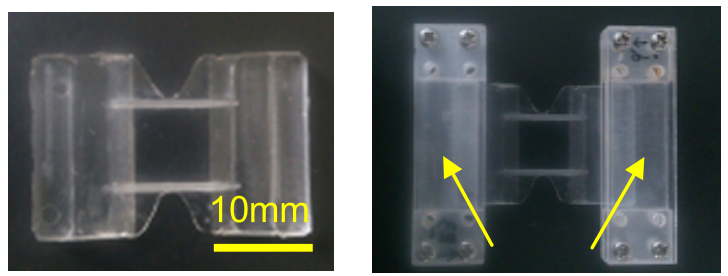

Fig. 1 Stretch chamber (A) and grippers (yellow arrows) attached to the chamber for connecting to stretching motors (B).

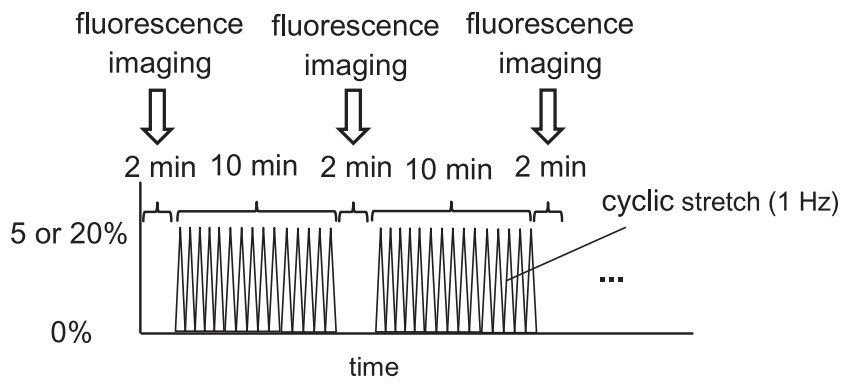

Fig. 2 Time course of cyclic stretch and fluorescence imaging. BAECs were subjected to cyclic stretch $(5 \%$ or $20 \%, 1 \mathrm{~Hz})$, which was paused for $2 \mathrm{~min}$ every $10 \mathrm{~min}$ for fluorescence imaging.

able for use with the fluorescence images obtained in this study because of low resolution. Thus, the fluorescence images were further analyzed using another software package, FibrilTool. This is a plugin of ImageJ developed by Boudaoud et al. [20] for analyzing the anisotropy and orientation of fibrous images. In this study, the lengths and alignments of mitochondria were estimated using the "anisotropy" and "orientation" analytical functions, respectively, of FibrilTool. For example, when the mitochondria are short, the anisotropy values are low, and vice versa. Since these interpreted values still contain some degree of uncertainty, the correlativity of these values was further evaluated using MicroP and FibrilTool (Fig. S1). The correlativity $(R)$ was 0.31 .

The cellular morphology, alignment and circularity were quantified with ImageJ as follows. A single cell was first outlined using the freehand function, and then approximate with ellipse. Finally, the ellipse, angle of the major axis, and circularity were quantified.

\subsection{Statistical analysis}

All behavioral data are expressed as mean \pm SEM. Data were analyzed using one-way analysis of variance (ANOVA), followed by Dunnett's test for post-hoc analysis to determine whether parameter values under $5 \%$ or $20 \%$ cyclic stretch were significantly different from those under $0 \%$ cyclic stretch (control).

\section{Results}

\subsection{Mitochondrial dynamics}

In BAECs under cyclic stretch, the morphology of mitochondria was sequentially imaged with time-lapse fluorescence imaging. Under 0\% (Movie S1) and 5\% cyclic stretch (Fig. 3A and Movie 


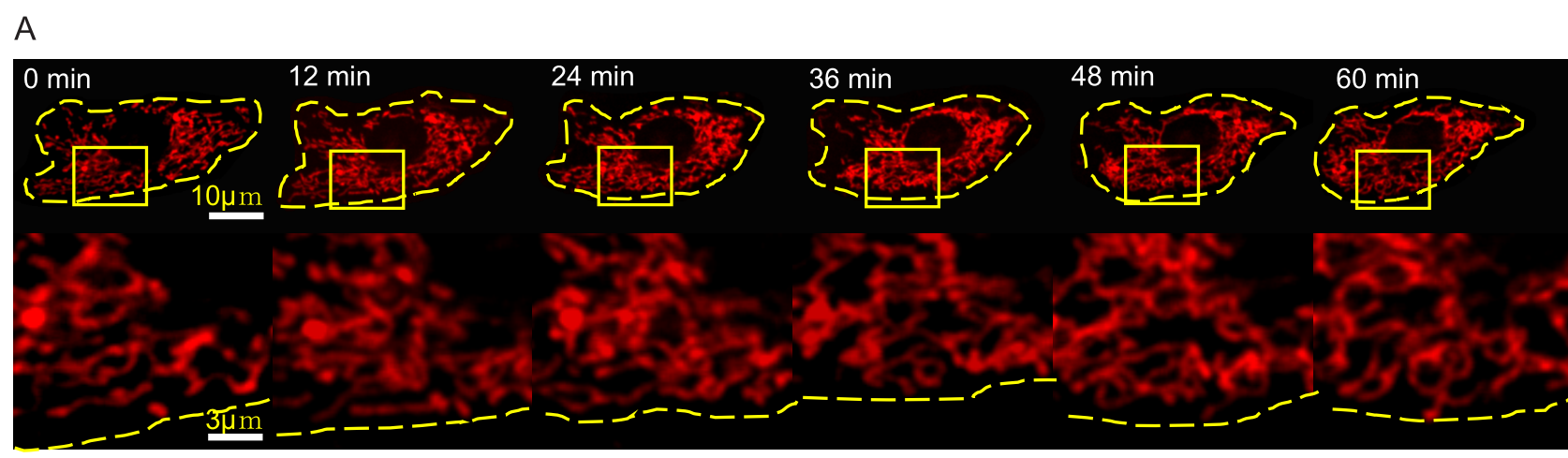

B

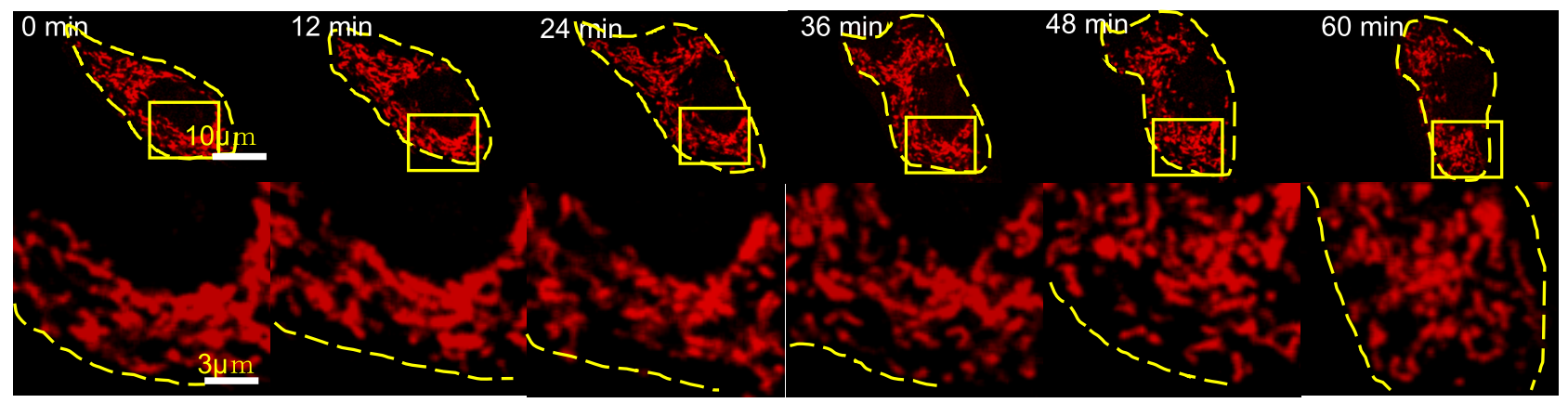

Fig. 3 Morphological dynamics of mitochondria under 5\% cyclic stretch (A) and 20\% cyclic stretch (B).

A

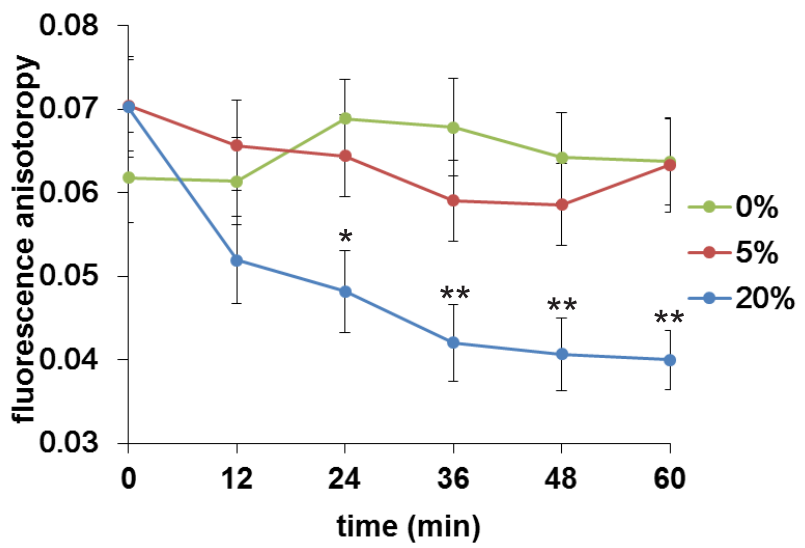

B

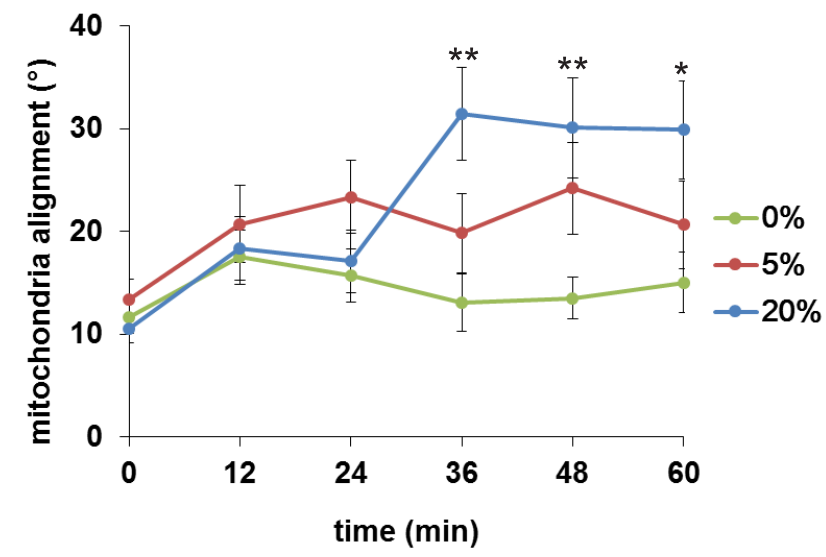

Fig. 4 Alterations in mitochondria morphology: mitochondria length estimated from fluorescence anisotropy (A) and mitochondrial alignment angle estimated from orientation (B). Data are expressed as mean \pm SEM ( $N=36$ for control, $N=28$ for $5 \%$ cyclic stretch, $n=30, N=30$ for $20 \%$ cyclic stretch). ${ }^{*} p<0.05$ vs. control, ${ }^{* *} p<0.01$ vs. control, one way ANOVA followed by Dunnett's test for post-hoc comparisons.

S2), the mesh morphology of mitochondria was preserved during the imaging period. On the other hand, under $20 \%$ cyclic stretch (Fig. 3B and Movie S3), the mesh morphology was disrupted. The mesh morphology gradually ruptured to exhibit rather sparse condensate. To quantitatively evaluate the morphological differences, the lengths and alignment angles of mitochondria were analyzed with image analysis.

The lengths of mitochondria were measured using two image-analysis tools: MicroP software that uses binary images, and FibrilTool that uses a spatial gradient of fluorescence intensity. Because the former version of MicroP was used to analyze the lengths of mitochondria in a previous study [19], we first used the latest version of MicroP to analyze the lengths of mitochondria in this study. We observed no distinct change in mean mitochondria length after 5\% cyclic stretch compared with $0 \%$ cyclic stretch, but detected a decrease in the mean length after $20 \%$ cyclic stretch (Fig. S2). Although analysis using MicroP showed a trend of change in mitochondrial length for $20 \%$ cyclic stretch, the results of the analysis of length alteration remain unclear. Consistent change in the mean length under $20 \%$ cyclic stretch was clearly captured using FibrilTool (Fig. 4A). While MicroP uses binary images, which is likely to be influenced significantly by fluorescence blur, the results obtained from FibrilTool may remain robust in the presence of such fluorescence blur because the soft- 
A

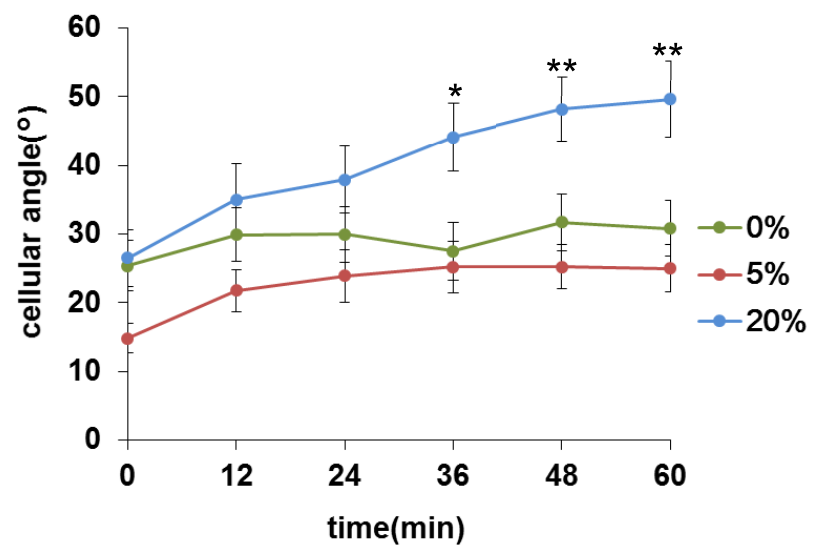

B

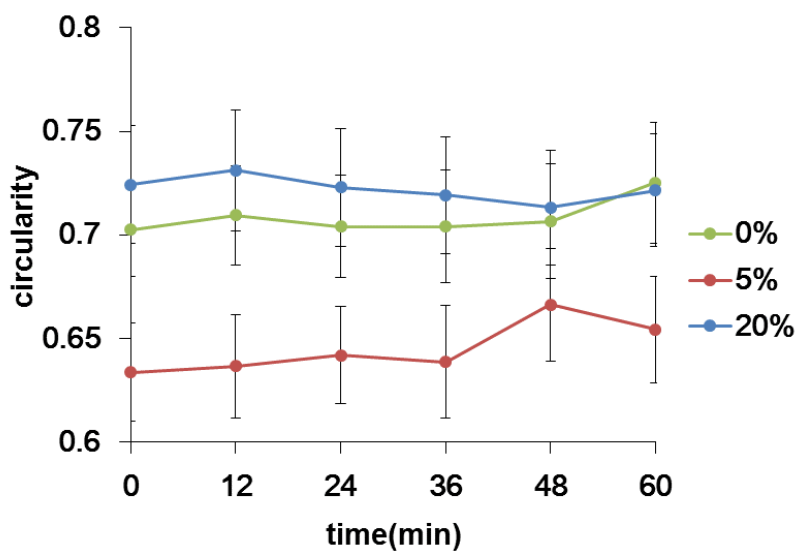

Fig. 5 Alteration in cellular morphology: cellular angle (A) and cellular circularity (B). Data are expressed as mean $\pm \mathrm{SEM}(\mathrm{N}=36$ for control, $\mathrm{N}=$ 28 for $5 \%$ cyclic stretch, $\mathrm{n}=30, \mathrm{~N}=30$ for $20 \%$ cyclic stretch). ${ }^{*} p<0.05$ vs. control, ${ }^{* *} p<0.01$ vs. control, one way ANOVA followed by Dunnett's test for post-hoc comparisons.

ware uses the gradient of the spatial distribution of fluorescence intensities for analysis.

The angle of mitochondrial alignment was also analyzed using FibrilTool. BAECs exhibited no difference in mean alignment angle under $5 \%$ cyclic stretch compared with $0 \%$ (control), while they exhibited a consistent increase in alignment angle under $20 \%$ cyclic stretch (Fig. 4B). This alteration in mitochondrial alignment was observed between 24 min and 36 min after the start of cyclic stretch. Since the reduction of mitochondrial length started within the first 12 min of cyclic stretch, it appeared that the mitochondria were first shortened and then underwent alignment change in BAECs under $20 \%$ cyclic stretch.

\subsection{Correlation with cellular alignment and circulation}

To examine the correlation between mitochondrial dynamics and cellular remodeling dynamics, cellular alignment and cellular circularity were quantified using the fluorescence images. No change in cellular alignment in BAECs was observed under 5\% cyclic stretch compared with control (Fig. 5A). Under 20\% cyclic stretch, however, cellular alignment was changed so that the BAECs were aligned perpendicular to the stretch axis. On the other hand, cellular circularity did not change when BAECs was subjected to $5 \%$ or $20 \%$ cyclic stretch compared with control (Fig. 5B). Thus, under 5\% cyclic stretch, BAECs exhibited no change in mitochondrial dynamics and no change in cellular remodeling dynamics. However, under $20 \%$ cyclic stretch, BAECs exhibited changes in both mitochondrial dynamics and cellular remodeling dynamics. These results indicate that cellular dynamics, especially changes in cellular alignment, may correlate with mitochondrial dynamics.

\section{Discussion}

In this study, mitochondrial dynamics were imaged with timelapse imaging under two conditions of cyclic stretch resulting in $5 \%$ and $20 \%$ elongation. The results suggested that the threshold of stretch that affects mitochondrial morphology under cyclic stretch lies between $5 \%$ and $20 \%$. In addition, our data showed that mitochondrial length as well as alignment angles of the cells and mitochondria were altered under $20 \%$ cyclic stretch in
BAECs, but not under 5\% cyclic stretch (Fig. 4, Fig. 5A). On the other hand, cellular circularity was not altered under both levels of cyclic stretch (Fig. 5B). Thus we concluded that in BAECs, mitochondrial morphology was altered under $20 \%$ cyclic stretch, in association with change in cellular alignment.

We speculate that shortening of mitochondria in BAECs under $20 \%$ cyclic stretch may involve the following two mechanisms.

First, fission of mitochondria may be enhanced by application of excessive cyclic stretch. Mitochondrial morphology is altered by repeated fission and fusion [10,11]. Our observations suggest that this balance is disrupted under excessive cyclic stretch, and fission overwhelms fusion to induce shortening of mitochondria. Although the biochemical mechanism in response to cyclic stretch involved in the overwhelming of fission is unknown, our observation supports the postulation that fission-enhancing proteins such as Drp1 could be activated. Drp1 is activated by the following mechanism. First, intracellular $\mathrm{Ca}^{2+}$ increases in cells under cyclic stretch [21]. When the level of intracellular $\mathrm{Ca}^{2+}$ is increased excessively, mitochondrial $\mathrm{Ca}^{2+}$ concentration also increases [22]. Increase in mitochondrial $\mathrm{Ca}^{2+}$ enhances the translocation of Drp1 to mitochondria in association with calcineurin [23] to activate Drp1.

Second, mitochondrial fission may be caused by cytoskeleton remodeling. Cyclic stretch stimulates remodeling of cytoskeletons and alters their alignment $[16,17]$. In this study, mitochondria were found to align perpendicularly to the stretch axis in response to $20 \%$ stretch (Fig. 4B). Because mitochondrial morphology is associated with microtubule morphology [13, 14], disruption of microtubules under cyclic stretch would disrupt mitochondrial morphology. It remains unclear whether or how far mitochondria are disrupted under microtubule remodeling. In addition to the mechanism, how that change affects cellular functions is not also clarified in the present study. Because the distribution of intracellular mitochondria affects the function of migrating cells [24], change in mitochondrial alignment may influence the functions of cells under cyclic stretch. In a previous study, when microtubules were disrupted by chemical stimulation, the mitochondria exhibited a rather twisted morphology as a 
result of release of mitochondria from microtubules [19]. If such twisting occurred in the present experiments, it might have been observed as shortening of mitochondrial length. However, it was difficult to distinguish fission from twisting on the fluorescence images in this study, because the quality of the images was insufficient.

Supra-physiological cyclic stretch has been suggested to induce ROS production $[5,6]$. However, whether the morphological dynamics of mitochondria as observed in this study are involved in ROS production remains uncertain. We observed shortening of mitochondria. We speculate that mitochondrial shortening may be associated with mitochondrial fission and mitochondrial release from microtubules, as discussed above. Because mitochondrial fission is known to enhance ROS production [12], by analogy mitochondrial shortening could also affect ROS production. On the other hand, the relationship between mitochondrial release from microtubules and ROS production is uncertain. Thus, it is unclear whether ROS production is enhanced in association with mitochondrial release. Enhancement of ROS production is associated with cellular apoptosis. Because cellular apoptosis induced by supra-physiological cyclic stretch appears to be involved in hypertension [2], understanding whether and how the mitochondrial dynamics observed under cyclic stretch are involved in apoptosis would be significant.

Although we observed both mitochondrial dynamics and cellular dynamics simultaneously in cells under cyclic stretch, it remains unclear whether the two are causally related. As a hypothesized causal relation, phosphorylation of focal adhesion kinase induced by mitochondrial ROS [7] may be involved in cell alignment alteration under cyclic stretch [25]. Further experiments, perhaps using inhibitors of mitochondrial and cellular dynamics, are required to understand whether the two are causally related or both depend on common causative factors. Moreover, this study observed no changes in cellular morphology in BAECs under $5 \%$ cyclic stretch. However, a previous study showed that cyclic stretch resulting in such small magnitude of elongation induced cellular dynamics [18]. If changes in cellular morphology can be controlled by varying the elongation value, the relationship between mitochondrial dynamics and cellular dynamics can be discussed more easily.

As discussed above, shortening of mitochondria could be associated with mitochondrial fission and release of mitochondria from microtubules. However, these phenomena could not be distinguished in this study. Various technical improvements are required to distinguish them clearly. First, clearer mitochondrial images should be obtained [19], and use of genetically encoded fluorescent proteins such as mitoYFP would be useful [26]. The signal-to-noise ratio of genetically encoded fluorescence proteins is suggested to be lower than that of chemically synthesized dyes. Second, as a means to further clarify mitochondrial dynamics, previous studies have analyzed the levels of photoactivated fluorescent proteins expressed in mitochondria [27, 28]. Using photoactivation, single mitochondria can be visualized independent of neighboring mitochondria. Through combined use of the above technical improvements, it should be possible to elucidate the morphological dynamics of mitochondria much more precisely.

In the present study, significant changes in mitochondrial morphology over time was observed when the cells were under $20 \%$ cyclic stretch, but no change was detected under $5 \%$ cyclic stretch. The result implies the presence of a threshold between $5 \%$ and 20\% stretch for alteration of mitochondrial morphology. Proof and identification of the threshold would help explain the mechanism of altered mitochondrial morphology under cyclic stretch, and facilitate planning of experiments that analyze intracellular signal activation under various magnitudes of cell elongation.

\section{Conclusion}

In this study, to examine whether mitochondrial morphology changes under cyclic stretch, mitochondria were time-lapse imaged in BAECs subjected to cyclic stretch that caused 5\% and $20 \%$ elongation. The results showed that the mitochondrial length was shortened and mitochondrial alignment was altered under $20 \%$ cyclic stretch. However, those morphological changes were not observed under $5 \%$ cyclic stretch. Furthermore, cellular alignment was altered when BAECs was subjected to $20 \%$ cyclic stretch, but not $5 \%$ cyclic stretch. These results suggest that mitochondrial morphology is altered in association with change in cellular alignment, and cyclic stretch of $20 \%$ affects mitochondrial dynamics different from cyclic stretch of $5 \%$.

\section{Acknowledgement}

This work was supported in part by a grant from JSPS KAKENHI Grant Number 25820017.

\section{Conflict of interest}

We have no conflicts of interest relationship with any companies or commercial organizations based on the definition of Japanese Society of Medical and Biological Engineering.

\section{References}

1. Califano JP, Reinhart-King CA: Exogenous and endogenous force regulation of endothelial cell behavior. J Biomech. 43(1), pp. 79-86, 2010.

2. Liu XM, Ensenat D, Wang H, Schafer AI, Durante W: Physiologic cyclic stretch inhibits apoptosis in vascular endothelium. FEBS Lett. 541(1-3), pp. 52-56, 2003.

3. Fujiwara K: Mechanical stresses keep endothelial cells healthy: beneficial effects of a physiological level of cyclic stretch on endothelial barrier function. Am J Physiol Lung Cell Mol Physiol. 285(4), L782-L784, 2003.

4. Zhang YH, Zhao CQ, Jiang LS, Dai LY: Cyclic stretch-induced apoptosis in rat annulus fibrosus cells is mediated in part by endoplasmic reticulum stress through nitric oxide production. Eur Spine J. 20(8), pp. 1233-1243, 2011.

5. Kou B, Zhang J, Singer DR.: Effects of cyclic strain on endothelial cell apoptosis and tubulogenesis are dependent on ROS production via $\mathrm{NAD}(\mathrm{P}) \mathrm{H}$ subunit p22phox. Microvasc Res. 77(2), pp. 125-133, 2009.

6. Sung HJ, Yee A, Eskin SG, McIntire LV: Cyclic strain and motion control produce opposite oxidative responses in two human endothelial cell types. Am J Physiol Cell Physiol. 293(1), C87C94, 2007.

7. Ali MH, Mungai PT, Schumacker PT: Stretch-induced phosphorylation of focal adhesion kinase in endothelial cells: role of mitochondrial oxidants. Am J Physiol Lung Cell Mol Physiol. 291(1), L38-L45, 2006.

8. Ali MH, Pearlstein DP, Mathieu CE, Schumacker PT: Mitochondrial requirement for endothelial responses to cyclic strain: im- 
plications for mechanotransduction. Am J Physiol Lung Cell Mol Physiol. 287(3), L486-L496, 2004.

9. Brookes PS, Yoon Y, Robotham JL, Anders MW, Sheu SS: Calcium, ATP, and ROS: a mitochondrial love-hate triangle. Am J Physiol Cell Physiol. 287, C817-C833, 2004.

10. Delivani P, Adrain C, Taylor RC, Duriez PJ, Martin SJ: Role for CED-9 and Egl-1 as regulators of mitochondrial fission and fusion dynamics. Mol Cell. 21(6), pp. 761-773, 2006.

11. Twig G, Hyde B, Shirihai OS: Mitochondrial fusion, fission and autophagy as a quality control axis: The bioenergetic view. Biochim Biophys Acta. 1777(9), pp. 1092-1097, 2008.

12. Yu T, Sheu SS, Robotham JL, Yoon Y: Mitochondrial fission mediates high glucose-induced cell death through elevated production of reactive oxygen species. Cardiovas Res. 79, pp. 341-351, 2008.

13. Frederick RL, Shaw JM: Moving mitochondria: establishing distribution of an essential organelle. Traffic. 8(12), pp. 1668-1675, 2007.

14. McCarron JG, Wilson C, Sandison ME, Olson ML, Girkin JM, Saunter C, Chalmers S: From structure to function: mitochondrial morphology, motion and shaping in vascular smooth muscle. J Vasc Res. 50(5), pp. 357-371, 2013.

15. Müller M, Mironov SL, Ivannikov MV, Schmidt J, Richter DW: Mitochondrial organization and motility probed by two-photon microscopy in cultured mouse brainstem neurons. Exp Cell Res. 303(1), pp. 114-127, 2005.

16. Mitsuoka Y, Tsukamoto A, Iwayoshi S, Furukawa KS, Ushida T: High time resolution time-lapse imaging reveals continuous existence and rotation of stress fibers under cyclic stretch in HUVEC. J Biomech Sci Eng. 7(2), pp. 188-198, 2012.

17. Morioka M, Parameswaran H, Naruse K, Kondo M, Sokabe M, Hasegawa Y, Suki B, Ito S: Microtubule dynamics regulate cyclic stretch-induced cell alignment in human airway smooth muscle cells. PLoS One. 6(10), e26384, 2011.

18. Kaunas R, Nguyen P, Usami S, Chien S: Cooperative effects of Rho and mechanical stretch on stress fiber organization. Proc Natl Acad Sci USA. 102(44), pp. 15895-15900, 2005.

19. Peng JY, Lin CC, Chen YJ, Kao LS, Liu YC, Chou CC, Huang YH, Chang FR, Wu YC, Tsai YS, Hsu CN: Automatic morphological subtyping reveals new roles of caspases in mitochondrial dynamics. PLoS Comp Biol. 7(10), e1002212, 2011.

20. Boudaoud A, Burian A, Borowska-Wykret D, Uyttewaal M, Wrzalik R, Kwiatkowska D, Hamant O: FibrilTool, an ImageJ plugin to quantify fibrillar structures in raw microscopy images. Nat Prot. 9(2), pp. 457-463, 2014.

21. Naruse K, Yamada T, Sokabe M.: Involvement of SA channels in orienting response of cultured endothelial cells to cyclic stretch. Am J Physiol Heart Circ Physiol. 274(5), H1532-H1538, 1998.

22. Kaddour-Djebbar I, Choudhary V, Brooks C, Ghazaly T, Lakshmikanthan V, Dong Z, Kumar MV: Specific mitochondrial calcium overload induces mitochondrial fission in prostate cancer cells. Int J Oncol. 36, pp. 1437-1444, 2010.

23. Cereghetti GM, Stangherlin A, Martins de Brito O, Chang CR, Blackstone C, Bernardi P, Scorrano L: Dephosphorylation by calcineurin regulates translocation of Drp1 to mitochondria. Proc Natl Acad Sci USA. 105(41), pp. 15803-15808, 2008.

24. Desai SP, Bhatia SN, Toner M, Irimia D: Mitochondrial localization and the persistent migration of epithelial cancer cells. Biophys J. 104(9), pp. 2077-2088, 2013.

25. Hsu HJ, Lee CF, Locke A, Vanderzyl SQ, Kaunas R: Stretch-induced stress fiber remodeling and the activations of JNK and ERK depend on mechanical strain rate, but not FAK. PLoS One.
5(8), e12470, 2010.

26. Kremneva E, Kislin M, Kang X, Khiroug L: Motility of astrocytic mitochondria is arrested by $\mathrm{Ca} 2+$-dependent interaction between mitochondria and actin filaments. Cell Calcium. 53(2), pp. 85-93, 2013.

27. Twig G, Graf SA, Wikstrom JD, Mohamed H, Haigh SE, Elorza A, Deutsch M, Zurgil N, Reynolds N, Shirihai OS: Tagging and tracking individual networks within a complex mitochondrial web with photoactivatable GFP. Am J Physiol Cell Physiol. 291(1), C176-C184, 2006.

28. Wikstrom JD, Katzman SM, Mohamed H, Twig G, Graf SA, Heart E, Molina AJA, Corkey BE, Moitoso de Vargas L, Danial NN, Collins S, Shirihai OS: Cell mitochondria exhibit membrane potential heterogeneity that can be altered by stimulatory or toxic fuel levels. Diabetes. 56(10), pp. 2569-2578, 2007.

\section{Aya Shinmura}

Aya SHINMura received the B.S. degree in Bioscience and Bioinformatics from Kyushu Institute of Technology, Fukuoka, Japan, in 2008. She is currently a graduate student of Graduate School of Engineering and Science at National Defense Academy, Kanagawa, Japan. Her research focus is

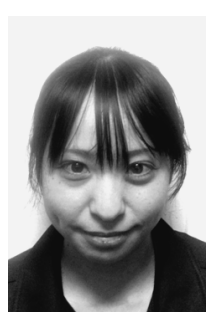
in the area of bioimaging of cellular functions and structures.

\section{Akira Tsukamoto}

Akira TsuкAмото received a Ph.D. in Engineering from The University of Tokyo in 2007 . He is presently assistant professor at School of Applied Sciences, National Defense Academy of Japan. His research interests are cellular biomechanics and mechanobiology. He is a member of Japanese So-

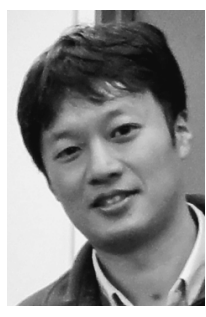
ciety for Medical and Biological Engineering and The Japan Society of Mechanical Engineers.

\section{Tsuyoshi HaMada}

Tsuyoshi HAMADA received the B.S. degree in Applied Physics from National Defense Academy, Kanagawa, Japan, in 2014. His research focus was in the area of bioimaging of cellular functions and structures.

\section{Kouki TaKemuRA}

Kouki TAKEMURA is currently a senior student in Department of Applied Physics, National Defense Academy, Kanagawa, Japan. His research focus is in the area of bioimaging of cellular functions and structures.
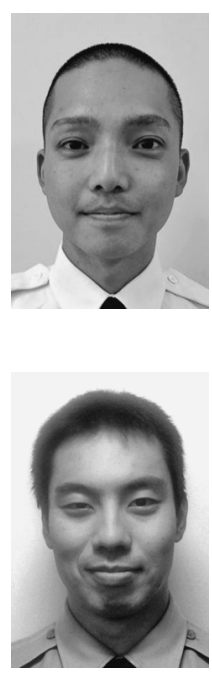


\section{Takashi UsHIDA}

Takashi UsHIDA received a Ph.D. in Engineering from The University of Tokyo in 1985. He is presently professor at School of Medicine, The University of Tokyo. His research interests are biomechanics and regenerated medicine. He is a member of Japanese Society for Medical and Biological

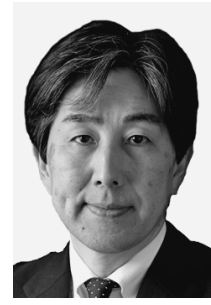
Engineering, Japan Society of Mechanical Engineers, Japanese Society for Regenerative Medicine, Japanese Society for Clinical Biomechanics.

\section{Shigeru TADA}

Shigeru TADA received the B.S., M.S. and Ph. D. degrees in mechanical engineering from Tokyo Institute of Technology, Tokyo, Japan, in 1987, 1989, 1992 respectively. He had been a research associate in Tokyo Institute of Technology from 1992 to 2007. He is currently a faculty member in the De-

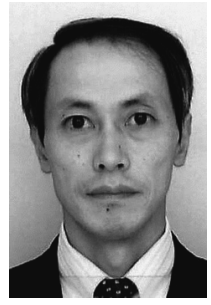
partment of Applied Physics at National Defense Academy, Kanagawa, Japan. His research focus is in the area of biomedical engineering and bio-fluid dynamic. 